\title{
Autophagy and autoimmunity crosstalks
}

\author{
Abhisek Bhattacharya and N. Tony Eissa* \\ Department of Medicine, Baylor College of Medicine, Houston, TX, USA
}

\section{Edited by:}

Christian Muenz, University of Zurich, Switzerland

\section{Reviewed by:}

Christian Muenz, University of Zurich, Switzerland

Willem Van Eden, Utrecht University, Netherlands

\section{${ }^{*}$ Correspondence}

N. Tony Eissa, Baylor College of

Medicine, One Baylor Plaza, BCM 285

Suite 535E, Houston, TX 77030, USA.

e-mail: teissa@bcm.edu
Autophagy, initially viewed as a conserved bulk-degradation mechanism, has emerged as a central player in a multitude of immune functions. Autophagy is important in host defense against intracellular and extracellular pathogens, metabolic syndromes, immune cell homeostasis, antigen processing and presentation, and maintenance of tolerance. The observation that the above processes are implicated in triggering or exacerbating autoimmunity raises the possibility that autophagy is involved in mediating autoimmune processes, either directly or as a consequence of innate or adaptive functions mediated by the pathway. Genome-wide association studies have shown association between single nucleotide polymorphisms (SNPs) in autophagy related gene 5 (Atg5), and Atg16/1 with susceptibility to systemic lupus erythematosus (SLE) and Crohn's disease, respectively. Enhanced expression of Atg5 was also reported in blood of mice with experimental autoimmune encephalomyelitis (EAE), a mouse model of multiple sclerosis (MS), and in $T$ cells isolated from blood or brain tissues from patients with active relapse of MS. This review explores the roles of autophagy pathway in the innate and adaptive immune systems on regulating or mediating the onset, progression, or exacerbation of autoimmune processes.

Keywords: autophagy, autophagosome, autoimmunity, encephalomyelitis, autoimmune, experimental, lupus erythematosus, systemic
The autophagy pathway, an evolutionary conserved mechanism, starts with the development of an isolation membrane within the cell that engulfs damaged organelles, misfolded proteins or pathogens, and eventually develops into an autophagosome. The autophagosomes, in turn, fuse with lysosomes to form the autophagolysosomes where the actual degradation of the substrates takes place (Levine et al., 2011). For the individual cell, the autophagy pathway is important not only to get rid of foreign or unwanted materials but also for efficient energy recycling during periods of stress. For the whole organism, the immune and physiological consequences of aberration of the autophagy pathway are much more profound. The immune system, responsible for surveillance and communication between different organs and cells types, is one such system in which the role of autophagy and the consequences of defects in autophagy go far beyond the degradative role of the pathway (Deretic, 2012a). Figure 1 shows potential roles of the autophagy pathway in the adaptive and innate immune systems that might modulate the onset and outcome of an autoimmune disease.

\section{AUTOPHAGY, THE ADAPTIVE IMMUNE SYSTEM AND AUTOIMMUNITY}

Autophagy plays important roles in both innate and adaptive immunity. Because there have been several excellent reviews on this topic (Munz, 2009; Sumpter and Levine, 2010; Kuballa et al., 2012; Randow and Munz, 2012), we will only discuss brief aspects of these roles as they might pertain to autoimmunity. Autophagy is essential for survival and homeostasis of lymphocytes and there exist at least two broad stages where autophagy might affect the adaptive immune cells. As the development of lymphocyte is a complex process involving inputs from other cells, both lymphocyte-intrinsic and extrinsic defects in autophagy might affect development and/or maturation of lymphocytes.

\section{AUTOPHAGY IN LYMPHOCYTE DEVELOPMENT}

$\mathrm{T}$ cell development in the thymus undergoes positive and negative selections, processes in which extrinsic inputs from thymic epithelial cells (TECs) play a major role in shaping the $\mathrm{T}$ cell repertoire. TECs show high levels of constitutive autophagy essential for proper display of MHC-antigen complex on their surface (Mizushima et al., 2004; Kasai et al., 2009), thereby facilitating appropriate $\mathrm{T}$ cell selection. Mice with $\operatorname{Atg} 5$ deficiency in TECs showed severely impaired central tolerance and autoimmune organ destruction, suggesting that autophagy-mediated display of MHC-antigen complex on surface of TECs is essential for proper T cell development (Nedjic et al., 2008). Autophagy deficiency in the TECs impaired both positive and negative selection mechanisms resulting into autoimmunity and it was proposed that autophagy-dependent display in the peripheral tissue needed to be counterbalanced by a similar tolerogenic mechanism in the thymus in order to prevent such autoimmune processes (Nedjic et al., 2008). Further, a recent report demonstrated the requirement of autophagy in TECs for loading endogenous antigens onto MHC-II and that this process was essential for negative selections of CD4 T cells (Aichinger et al., 2013). Because both DCs and TECs might be important in differentiation of regulatory $\mathrm{T}$ cells (Tregs) (Wirnsberger et al., 2009; Hinterberger et al., 2010), this report suggested that autophagy might be important in differentiation of Tregs (Aichinger et al., 2013). As Tregs are among the major players controlling autoimmunity (La Cava, 2009), this might 


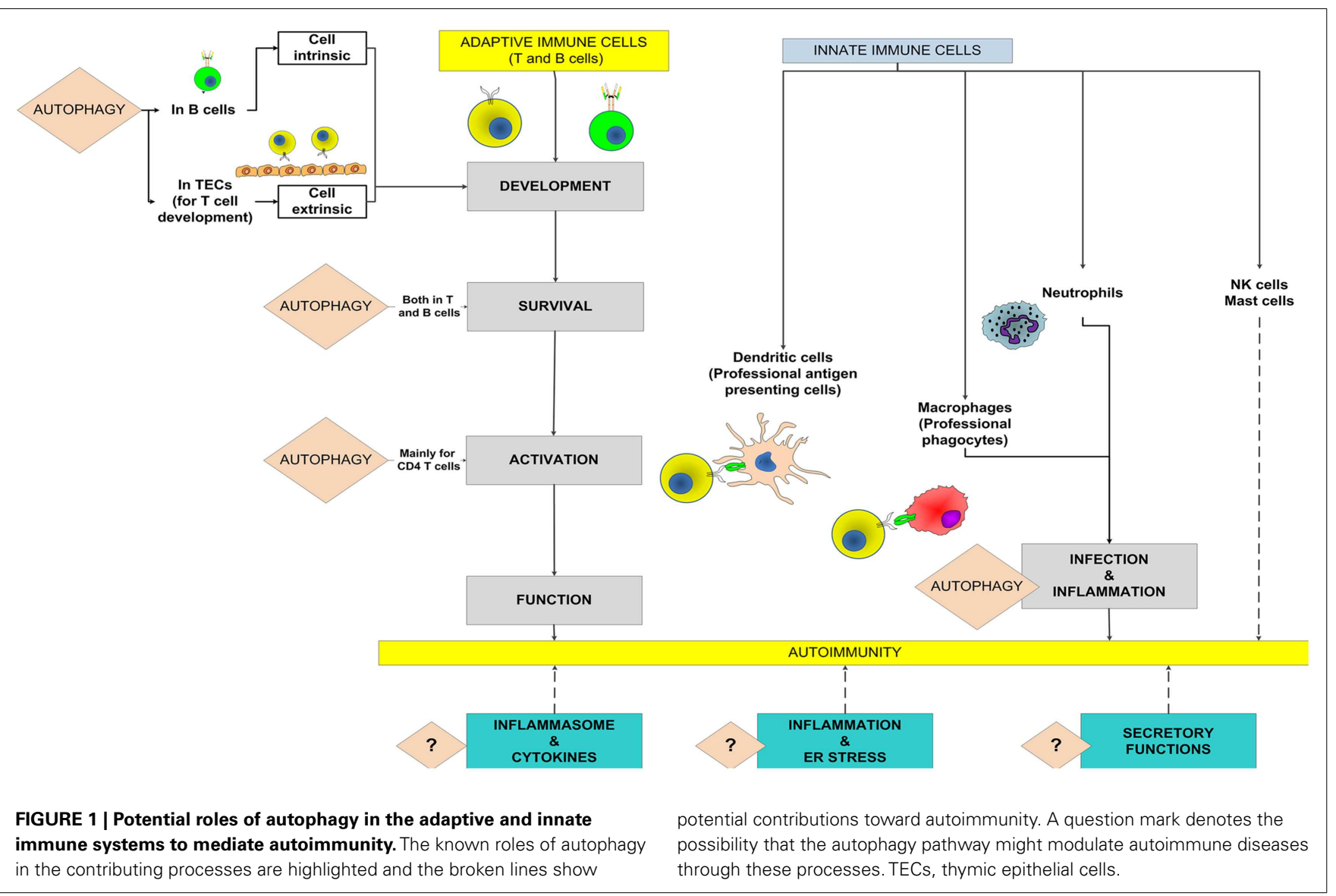

be another potential link between autophagy and autoimmune diseases.

Fetal liver chimera and conditional knock-out studies have shown that $\mathrm{T}$ cell development remained normal in mice lacking Atg5 in T cells but peripheral T cell compartment showed reduction in numbers, particularly in CD8 T cells (Pua et al., 2007). These results were attributed to the pro-survival role of autophagy in mature T cells. Studies showed considerable interaction between the autophagy and apoptotic pathways (Maiuri et al., 2007). Atg3, 5, or 7-deficient mature $\mathrm{T}$ cells showed defective Endoplasmic reticulum (ER) homeostasis and mitochondrial clearance and, consequently, an elevated levels of ROS, which might serve as one of the potential links between the autophagy and apoptotic pathways (Pua et al., 2007; Jia and He, 2011; Jia et al., 2011). However, increased levels of mitochondria were observed in Atg7-/- but not in Atg5-/ - thymocytes at the single positive stage (Pua et al., 2009). A possible explanation could be different stages or extent of involvement of these proteins in mitochondrial clearance. These findings potentially brings another layer of complexity into focus, namely autophagy-independent effect of various Atg.

In contrast to T cells, autophagy in B cells plays a very important role in development and the requirement of Atg5 has been found to be highly stage-specific, with a defective pro- to pre-B cell transition in B cell-specific Atg5-/- knock-out mice. In these mice, the levels of pre and immature B cells, along with peritoneal B1 cells were reduced to a great extent. This finding was also attributed to a role of autophagy in maintaining B cell survival (Miller et al., 2008).

\section{AUTOPHAGY IN LYMPHOCYTE FUNCTIONS}

Autophagy induction in response to starvation and TCR stimulation has been observed in mouse T cells (Pua et al., 2007) and in cultured human $\mathrm{T}$ cells during aging (Gerland et al., 2004) and in HIV infection (Espert et al., 2006). Atg5-deficient $\mathrm{T}$ cells showed reduced proliferation upon both TCR and PMAionomycin stimulation (Pua et al., 2007). This finding highlights potentially different roles of autophagy in naïve versus activated $\mathrm{T}$ cells. Most studies involving autophagy in $\mathrm{T}$ cells focused on roles of autophagy in cell survival and found autophagy to be a pro-survival mechanism (Pua et al., 2007). However, some studies have also suggested that autophagy might be required for $\mathrm{T}$ cell death (Espert et al., 2006; Bell et al., 2008). Uninfected lymphocytes undergo autophagy-mediated cell death upon engagement of the receptor CXCR4 by HIV envelop glycoprotein (Espert et al., 2006). Another study has also shown that autophagy could be an important cell death machinery in T cells lacking caspase-8 or Fas-associated death domain (FADD) activity, thereby raising the possibility that interaction between autophagy and apoptosis might be context dependent (Bell et al., 2008). It is possible that, in activated $\mathrm{T}$ cells, autophagy plays different roles compared to naïve cells and might be involved in activation-induced cell death following $\mathrm{T}$ cell proliferation in immune response. 
Recent findings suggest that autophagy might affect overall $\mathrm{T}$ cell functions under different conditions of polarization and activation. Rapamycin, an mTOR inhibitor and inducer of autophagy, has been found to promote $\mathrm{T}$ cell memory when administered in low doses, although it is not clear if this effect is mediated by autophagy (Araki et al., 2009). Moreover, low dose rapamycin exacerbated autoimmune experimental uveitis, and this action of rapamycin was thought to be mediated by autophagy (Zhang et al., 2012). Interestingly, expression of Atg5 has been shown to correlate with severity of experimental autoimmune encephalitis (EAE), a mouse model of multiple sclerosis (MS), and to be increased in T cells of MS patients during relapses (Alirezaei et al., 2009), which can worsen by prolonged autoreactive $\mathrm{T}$ cell survival. EAE is considered predominantly a CD4 mediated disease and further studies are required to dissect how autophagy in $\mathrm{T}$ cells influences the onset or progression of autoimmune diseases in animal models and if these roles of autophagy are also dependent on cell survival. These studies can develop in vivo models in which the roles of autophagy in CD4 or CD8 cells could be studied independent to its pro- or anti-survival functions, particularly in the context of an infection or autoimmune disease.

In mature B cells, BCR signal can lead to B cell activation or apoptosis, depending on the context. Autophagy is involved in both processes, with BCR-activation-mediated cell death being associated with extensive autophagosome formation (Watanabe et al., 2008). B cells are capable of antigen processing following BCR ligation and autophagy might be involved in such process (Watanabe et al., 2008). BCR signaling recruits TLR-9 to autophagosome for further interaction with its ligand (Chaturvedi et al., 2008). Systemic lupus erythematosus (SLE) is perhaps the most studied autoimmune disease with respect to the roles of autophagy in autoimmune processes (Pierdominici et al., 2012). There are a number of potential mechanisms by which autophagy might influence the pathogenesis of SLE, modulating both the adaptive and innate immune system. As B cells represent a major player in SLE, in which they act by both antibody-dependent and antibody-independent mechanisms, autophagy-mediated B cell modulation might directly influence the pathophysiology of SLE.

\section{AUTOPHAGY, THE INNATE IMMUNE SYSTEM AND AUTOIMIMUNITY}

Autoimmunity results from uncontrolled action of the adaptive immune cells, however, activation of the adaptive system depends on the innate immune cells and the innate immune system is perhaps the most extensively studied component with respect to the role of autophagy in shaping the organization and functions of the system (Deretic, 2012b).

The innate immune functions can be broadly categorized into four overlapping stages, migration, recognition and phagocytosis, antigen processing and presentation, and cytokine secretion. Autophagy plays particularly important roles in the last three stages, thereby not only shaping the innate immune response but also influencing the activation of the adaptive immune compartment.

\section{LEVEL ONE: PHAGOCYTOSIS, AUTOPHAGY, AND AUTOIMMUNITY}

The role of autophagy in innate immunity is best characterized with respect to pathogen elimination. Both pathogen recognition and intracellular killing can be controlled by autophagy. The autophagy pathway interacts extensively with a number of pattern recognition receptors $(\mathrm{PRR})$ and $\mathrm{PRR}$ activation in a wide variety of cases has been shown to induce autophagy (Tang et al., 2012). However, recent evidences suggest that this process might also extend beyond pathogen control.

Phagocytosis can be viewed as a coordinated interaction between two different kinds of players, a predator that engulfs the materials to be cleared, macrophages being the professional phagocytes in the body, and prey to be engulfed such as a pathogen, foreign materials, or dead cells. Autophagy has been found to be an essential process for dead cell clearance. Apoptotic cells release lysophosphatidylcholine (LPC) as a "come-get-me" signal for phagocytes and upregulate phosphatidylserine (PS) as an "eat me" signal on their surface. Autophagy genes are essential for efficient release of LPC and in absence of autophagy, apoptotic cells fail to express PS properly on their surface (Qu et al., 2007).

On the other hand, proteins involved in autophagy pathway, such as LC3-II (Microtubule-associated protein 1 light chain 3 alpha), Beclin 1, and VPS34, are recruited to phagosomes following phagocytosis of particles containing TLR ligands by macrophages (Sanjuan et al., 2007). LC3-associated phagocytosis, a process distinct from classical autophagy, has also been found to be necessary to carry out efficient dead cell clearance (Martinez et al., 2011) and defects in expression of MARCO (macrophage receptor with collagenous structure), a receptor involved in dead cell clearance, has been shown to result into reduced dead cell clearance and SLE in mice (Rogers et al., 2009). Thus, an absence of autophagy or autophagic proteins might result into defective clearance of apoptotic cells. As defects in apoptotic cell clearance have been linked to a number of autoimmune diseases, such as SLE, it is possible that autophagy might modulate the susceptibility to autoimmunity.

It should also be noted that autophagy induction in macrophages has been shown to affect phagocytosis of pathogens, though the reports are conflicting, indicating both increase and decrease in phagocytosis following induction of autophagy (Martinet et al., 2009; Lima et al., 2011). A number of autoimmune diseases are precipitated or exacerbated following infection (Kivity et al., 2009). It would be important to determine if changes in apoptotic cell clearance occur following infection-induced modulation in autophagy, which in turn could modulate the induction or exacerbation of autoimmune processes.

\section{LEVEL TWO: ANTIGEN PRESENTATION, AUTOPHAGY, AND AUTOIMMUNITY}

The classic definition of antigen presentation is that extracellular antigens are presented in the context of class II MHC following endocytosis and phagolysosomal degradation (Gannage and Munz, 2010). Recent evidence suggests that this process depends on the autophagy pathway. Characterization of the MHC-II ligands, called ligandome, in a human B lymphoblastoid cell line showed that peptides from intracellular sources are presented on 
MHC-II and starvation-induced autophagy enhanced this process (Dengjel et al., 2005). Further, autophagosomes colocalize with MHC-II loading compartments in two important antigen presenting cells (APCs) that shape up the entire adaptive immune repertoire. These cells are TECs, that shape up the T cell repertoire, and the dendritic cells (DC) that act as the professional APCs (Schmid and Munz, 2007; Schmid et al., 2007). Mice with DCs lacking Atg5 succumbed to HSV-2 infection and showed defective CD4 T cell priming. These DCs showed defective antigen presentation resulting from a profound defect in processing and delivery of antigens containing TLR ligands to MHC-II compartment and delayed phagolysosomal fusion and degradation of the antigens (Lee et al., 2010). Autophagy induction in bone-marrow DCs also enhanced presentation of mycobacterial antigen and mice immunized with rapamycin-treated DCs showed stronger $\mathrm{T}$ cell response upon challenge with Mtb (Jagannath et al., 2009). Autophagy in APCs is involved in presentation of citrullinated peptide, a hallmark of rheumatoid arthritis, in context of class II MHC (Ireland and Unanue, 2011). It also has been suggested that autophagy may be involved in class I antigen presentation to CD8 T cells, particularly in context of viral infection (English et al., 2009; Uhl et al., 2009).

Dendritic cell-mediated antigen presentation in the context of MHC-II is perhaps an area where the role of autophagy could directly influence autoimmune diseases. Activation of the adaptive immune cells, the major players in most autoimmune diseases, depends primarily on DC-mediated antigen presentation. Genome-wide association studies have identified Atg5 as one of the susceptibility loci in SLE (Harley et al., 2008; Gateva et al., 2009; Han et al., 2009; Zhou et al., 2011a), though the functional significance of this finding is yet to be established. A number of possibilities have been raised ranging from increased survival of pathogenic $\mathrm{T}$ cells to defects in apoptotic cell clearance and several autophagy modulators are currently in clinical trials for SLE (Pierdominici et al., 2012). It is interesting to note that the PRDM1-ATG5 intergenic region has also been associated with susceptibility to rheumatoid arthritis (Raychaudhuri et al., 2009) and a common role of the autophagy pathway in different autoimmune diseases has been proposed (Zhou et al., 2011a). Another autophagy gene associated with autoimmunity is Atg16l1, being implicated in Crohn's disease (CD) (Parkes et al., 2007). DCs with patients of CD, harboring particular Atg16l1 risk variant, showed defects in autophagy induction and in presentation and priming of pathogen-specific CD4 T cells (Cooney et al., 2010). Interaction between the gut microflora and the mucosal immune system plays a pivotal role in CD (Manichanh et al., 2012) and autophagy in mucosal immune cells might also influence the pathophysiology and outcome of CD. Indeed, a recent report showed that an intact autophagy pathway restricted intracellular replication of adherent-invasive Escherichia coli, implicated in the pathogenesis of CD; without affecting the replication of other commensal or pathogenic strains of E. coli involved in gastroenteritis (Lapaquette et al., 2010).

It would be informative to determine the phenotype of mice with autophagy deficiency in DCs, in autoimmune disease models of MS, a predominantly CD4 $\mathrm{T}$ cell mediated disease, or rheumatoid arthritis.

\section{LEVEL THREE: CYTOKINES, ER STRESS, AUTOPHAGY, AND AUTOIMMUNITY}

The third important link between autophagy and autoimmunity could be through modulating cytokine secretion, particularly in the context of inflammasome activation. Autophagy plays a negative role with respect to inflammasome activation and autophagy deficiency leads to increased production of IL-1 $\beta$ and IL-18 (Nakahira et al., 2011; Zhou et al., 2011b). Diseases resulting from increased activation of the immune system comprise two different categories: autoinflammatory diseases, characterized by inflammation mediated predominantly by innate immune cells, including macrophages and neutrophils, and autoimmune diseases in which the adaptive immune cells target the self-antigens (McGonagle et al., 2009). The inflammasome-mediated effects belong to the former category and the role of inflammasomes in these diseases has been reviewed (Shaw et al., 2011). However, given the extensive effects of IL- $1 \beta$ on adaptive immune cells, autophagy might also affect the outcome of autoimmune diseases by modulating IL- $1 \beta$ production. As a whole, IL- $1 \beta$ and IL-18 enhance the functional responses of $\mathrm{B}$ and $\mathrm{T}$ cells including IL-2 receptor expression and lifespan, antibody production by $\mathrm{B}$ cells, and $\mathrm{T}_{\mathrm{H}} 1$ and $\mathrm{T}_{\mathrm{H}} 17$ polarization effects (Ben-Sasson et al., 2009; Chung et al., 2009). Thus IL-1 $\beta$ and IL-18 might well serve as a bridge between autophagy in innate cells and the adaptive immune response. In this context, IL- $1 \beta$ receptor blockade had beneficial effects in rheumatoid arthritis and has been suggested as a therapy for autoinflammatory diseases (Goldbach-Mansky, 2009). Conflicting reports exist regarding the role of inflammasome activation in EAE, with one study showing roles of NLRP3 inflammasome in EAE progression (Gris et al., 2010), whereas another study found no such role but reported an inflammasome-independent role of ASC (Shaw et al., 2010). The gut microbiota have important roles in shaping the immune system as a whole and particularly in models of MS (Ivanov et al., 2009; Ochoa-Reparaz et al., 2009; Berer et al., 2011). Given the extensive interaction between autophagy and different microbes, it would be informative to determine how autophagy and gut microflora interact to influence autoimmune diseases.

Recent evidence showed that autophagy played an important role in pancreatic beta cell functions and might modulate glucose homeostasis as a whole (Ebato et al., 2008; Jung et al., 2008). ER stress has an important role in the pathogenesis of diabetes and autophagy plays a role in this process as well (Quan et al., 2012). Since ER stress is involved in insulin resistance (Ozcan et al., 2006), autophagy might also be involved in insulin resistance by modulating ER stress response.

\section{LEVEL FOUR: SECRETION, AUTOPHAGY, AND AUTOIMMUNITY}

Secretion from cells can proceed through two broad pathways: a well-characterized canonical pathway in which proteins with a signal peptide go through ER and Golgi. However, secretion of proteins without a signal peptide proceeds through an ER-Golgi independent pathway. Interestingly, it was proposed that secretion of such proteins might, in part, be mediated by autophagy (Giuliani et al., 2011). Autophagy-mediated secretion of acyl coenzyme A (CoA) binding protein (ACBP), a cytosolic protein 
without a signal peptide, was reported in yeasts (Duran et al., 2010; Manjithaya et al., 2010) and recent reports also suggested that autophagy is involved in a number of secretory processes in immune and non-immune cells. Autophagy modulates secretory processes in the context of osteoclastic bone formation (DeSelm et al., 2011), from mast cells (Ushio et al., 2011), intestinal Paneth cells (Cadwell et al., 2008), presynaptic neurotransmission (Hernandez et al., 2012), and secretion of IL-1 $\beta$ (Dupont et al., 2011). Though the relationship between autophagy and IL- $1 \beta$ secretion is complicated owing to the fact that autophagy inhibits inflammasome activation (Nakahira et al., 2011), a recent report showed that baseline autophagy inhibits IL-1 $\beta$ secretion whereas induced autophagy increases secretion of IL-1 $\beta$ (Dupont et al., 2011).

Elevated levels of type I interferon, interferon-alpha (IFN- $\alpha$ ) being the prototypic one, is the hallmark of SLE and clinical trials are going on with monoclonal antibodies against IFN- $\alpha$ in SLE (Lichtman et al., 2012). Interestingly, autophagy is also involved in type I IFN secretion. Autophagy is required in plasmacytoid dendritic cells ( $\mathrm{pDCs}$ ), a major source of IFN- $\alpha$, for sensing ssRNA virus and secretion of IFN- $\alpha$ (Lee et al., 2007). Similarly mTOR inhibition has also been shown to reduce IFN- $\alpha$ secretion by pDCs in response to TLR-9 ligands (Cao et al., 2008), though whether this is mediated by autophagy remains to be elucidated. However, in contrast to pDCs which use Toll-like receptor 7 (TLR7) for sensing ssRNA viruses, most other cell types in the body use cytosolic RNA sensors such as RIG-I and MDA-5, belonging to the RLR family, for this purpose. Atg5 deficiency in MEF has been shown to increase IFN- $\alpha$ secretion in context of viral infections by suppressing RLR signaling (Jounai et al., 2007; Tal et al., 2009). This finding represents a non-canonical role of Atg5 (Takeshita et al., 2008; Tal et al., 2009). Non-canonical autophagy was also shown to mediate IFN- $\alpha$ secretion in response to DNA-immune complex (Henault et al., 2012). Thus, modulation of IFN- $\alpha$ secretion by autophagy pathway might play a role in SLE. In a recent study, analysis of SLE metabolome in serum samples of SLE patients showed increased

\section{REFERENCES}

Aichinger, M., Wu, C., Nedjic, J., and Klein, L. (2013). Macroautophagy substrates are loaded onto MHC class II of medullary thymic epithelial cells for central tolerance. J. Exp. Med. 210, 287-300.

Alirezaei, M., Fox, H. S., Flynn, C. T., Moore, C. S., Hebb, A. L., Frausto, R. F., et al. (2009). Elevated ATG5 expression in autoimmune demyelination and multiple sclerosis. Autophagy 5, 152-158.

Araki, K., Turner, A. P., Shaffer, V. O., Gangappa, S., Keller, S. A., Bachmann, M. F., et al. (2009). mTOR regulates memory CD8 T-cell differentiation. Nature 460, 108-112.

Bell, B. D., Leverrier, S., Weist, B. M., Newton, R. H., Arechiga, A. F., Luhrs, K. A., et al. (2008). FADD and caspase- 8 control the outcome of autophagic signaling in proliferating

oxidative stress (Wu et al., 2012). Autophagy deficiency is generally associated with increased oxidative stress secondary to accumulation of damaged mitochondria (Zhou et al., 2011b). On the other hand, autophagy inhibition leads to accumulation of p62 (Mizushima and Komatsu, 2011) which, in turn, activates Nrf2 (nuclear factor erythroid 2-related factor 2) (Komatsu et al., 2007). $\mathrm{Nrf} 2$ works as a major player in the oxidative stress response pathway (Kaspar et al., 2009). The effect of modulation of autophagy on oxidative stress of SLE warrants further studies.

It would be interesting to test how autophagy-mediated secretory functions influence autoimmune processes. Though considered to be mediated by adaptive immune cells, autoimmune processes, as in MS, are influenced by innate immune cells (Gandhi et al., 2010). The role of autophagy in secretion might have added significance in cells such as NK cells and neutrophils, which function mainly through secretion and degranulation. Another important area for future exploration would be the role of autophagy in myeloid-derived suppressor cells that suppress $\mathrm{T}$ cell function.

\section{CONCLUSION}

Given the above potential implications of autophagy in autoimmunity, it is rather surprising that there are only few in vivo reports on the functional correlation between autophagy and autoimmune diseases. Non-specific autophagy-lysosomal inhibitors, such as chloroquine, have long been used in clinics to treat SLE and rheumatoid arthritis (He et al., 2011). It is essential to understand the complex interplay between autophagy and autoimmunity in order to develop effective and more specific therapeutic strategies. Autophagy might play different roles in an autoimmune disease depending on the cell types involved and thus the ultimate results of pharmacological modulation might depend on the downstream effector involved. Given the paucity of in vivo data, it will be important to determine how the findings from animal models translate to human conditions, as pathophysiology of autoimmune diseases vary considerably between humans and lower animals.

Murthy, N., et al. (2008). Tolllike receptor-mediated induction of type I interferon in plasmacytoid dendritic cells requires the rapamycin-sensitive $\mathrm{PI}(3) \mathrm{K}$-mTORp70S6K pathway. Nat. Immunol. 9, 1157-1164.

Chaturvedi, A., Dorward, D., and Pierce, S. K. (2008). The B cell receptor governs the subcellular location of Toll-like receptor 9 leading to hyperresponses to DNAcontaining antigens. Immunity 28, 799-809.

Chung, Y., Chang, S. H., Martinez, G. J., Yang, X. O., Nurieva, R., Kang, H. S., et al. (2009). Critical regulation of early Th17 cell differentiation by interleukin-1 signaling. Immunity 30, 576-587.

Cooney, R., Baker, J., Brain, O., Danis, B., Pichulik, T., Allan, P., et al. (2010). NOD2 stimulation induces autophagy in dendritic cells influencing bacterial handling and antigen presentation. Nat. Med. 16, 90-97.

Dengjel, J., Schoor, O., Fischer, R., Reich, M., Kraus, M., Muller, M., et al. (2005). Autophagy promotes MHC class II presentation of peptides from intracellular source proteins. Proc. Natl. Acad. Sci. U.S.A. 102, 7922-7927.

Deretic, V. (2012a). Autophagy: an emerging immunological paradigm. J. Immunol. 189, 15-20.

Deretic, V. (2012b). Autophagy as an innate immunity paradigm: expanding the scope and repertoire of pattern recognition receptors. Curr. Opin. Immunol. 24, 21-31.

DeSelm, C. J., Miller, B. C., Zou, W., Beatty, W. L., van Meel, E., Takahata, Y., et al. (2011). Autophagy proteins regulate the secretory component of osteoclastic bone resorption. Dev. Cell 21, 966-974. 
Dupont, N., Jiang, S., Pilli, M., Ornatowski, W., Bhattacharya, D., and Deretic, V. (2011). Autophagy-based unconventional secretory pathway for extracellular delivery of IL-1beta. EMBO J. 30, 4701-4711.

Duran, J. M., Anjard, C., Stefan, C., Loomis, W. F., and Malhotra, V. (2010). Unconventional secretion of Acb1 is mediated by autophagosomes. J. Cell Biol. 188, 527-536.

Ebato, C., Uchida, T., Arakawa, M., Komatsu, M., Ueno, T., Komiya, K., et al. (2008). Autophagy is important in islet homeostasis and compensatory increase of beta cell mass in response to high-fat diet. Cell Metab. 8, 325-332.

English, L., Chemali, M., Duron, J., Rondeau, C., Laplante, A., Gingras, D., et al. (2009). Autophagy enhances the presentation of endogenous viral antigens on MHC class I molecules during HSV-1 infection. Nat. Immunol. 10, 480-487.

Espert, L., Denizot, M., Grimaldi, M., Robert-Hebmann, V., Gay, B., Varbanov, M., et al. (2006). Autophagy is involved in $\mathrm{T}$ cell death after binding of HIV-1 envelope proteins to CXCR4. J. Clin. Invest. 116, 2161-2172.

Gandhi, R., Laroni, A., and Weiner, H. L. (2010). Role of the innate immune system in the pathogenesis of multiple sclerosis. J. Neuroimmunol. 221, 7-14.

Gannage, M., and Munz, C. (2010). MHC presentation via autophagy and how viruses escape from it. Semin. Immunopathol. 32, 373-381.

Gateva, V., Sandling, J. K., Hom, G., Taylor, K. E., Chung, S. A., Sun, X., et al. (2009). A large-scale replication study identifies TNIP1, PRDM1, JAZF1, UHRF1BP1 and IL10 as risk loci for systemic lupus erythematosus. Nat. Genet. 41, 1228-1233.

Gerland, L. M., Genestier, L., Peyrol, S., Michallet, M. C., Hayette, S., Urbanowicz, I., et al. (2004). Autolysosomes accumulate during in vitro CD8 + T-lymphocyte aging and may participate in induced death sensitization of senescent cells. Exp. Gerontol. 39, 789-800.

Giuliani, F., Grieve, A., and Rabouille, C. (2011). Unconventional secretion: a stress on GRASP. Curr. Opin. Cell Biol. 23, 498-504.

Goldbach-Mansky, R. (2009). Blocking interleukin-1 in rheumatic diseases. Ann. N. Y. Acad. Sci. 1182, 111-123.

Gris, D., Ye, Z., Iocca, H. A., Wen, H., Craven, R. R., Gris, P., et al. (2010). NLRP3 plays a critical role in the development of experimental autoimmune encephalomyelitis by mediating Th1 and Th17 responses. J. Immunol. 185, 974-981.

Han, J. W., Zheng, H. F., Cui, Y., Sun, L. D., Ye, D. Q., Hu, Z., et al. (2009). Genome-wide association study in a Chinese Han population identifies nine new susceptibility loci for systemic lupus erythematosus. Nat. Genet. 41, 1234-1237.

Harley, J. B., Alarcon-Riquelme, M. E., Criswell, L. A., Jacob, C. O., Kimberly, R. P., Moser, K. L., et al. (2008). Genome-wide association scan in women with systemic lupus erythematosus identifies susceptibility variants in ITGAM, PXK, KIAA1542 and other loci. Nat. Genet. 40, 204-210.

He, Y., Xu, Y., Zhang, C., Gao, X., Dykema, K. J., Martin, K. R., et al. (2011). Identification of a lysosomal pathway that modulates glucocorticoid signaling and the inflammatory response. Sci. Signal. 4, ra44.

Henault, J., Martinez, J., Riggs, J. M., Tian, J., Mehta, P., Clarke, L., et al. (2012). Noncanonical autophagy is required for type I interferon secretion in response to DNA-immune complexes. Immunity 37, 986-997.

Hernandez, D., Torres, C. A., Setlik, W., Cebrian, C., Mosharov, E. V., Tang, G., et al. (2012). Regulation of presynaptic neurotransmission by macroautophagy. Neuron 74 , 277-284.

Hinterberger, M., Aichinger, M., Prazeres da Costa, O., Voehringer, D., Hoffmann, R., and Klein, L. (2010). Autonomous role of medullary thymic epithelial cells in central CD4(+) $\mathrm{T}$ cell tolerance. Nat. Immunol. 11, 512-519.

Ireland, J. M., and Unanue, E. R. (2011). Autophagy in antigen-presenting cells results in presentation of citrullinated peptides to CD4 T cells. J. Exp. Med. 208, 2625-2632.

Ivanov, I. I., Atarashi, K., Manel, N., Brodie, E. L., Shima, T., Karaoz, U., et al. (2009). Induction of intestinal Th 17 cells by segmented filamentous bacteria. Cell 139, 485-498.

Jagannath, C., Lindsey, D. R., Dhandayuthapani, S., Xu, Y., Hunter, R. L. Jr., and Eissa, N. T. (2009). Autophagy enhances the efficacy of BCG vaccine by increasing peptide presentation in mouse dendritic cells. Nat. Med. 15, 267-276.

Jia, W., and He, Y. W. (2011). Temporal regulation of intracellular organelle homeostasis in T lymphocytes by autophagy. J. Immunol. 186, 5313-5322.

Jia, W., Pua, H. H., Li, Q. J., and He, Y. W. (2011). Autophagy regulates endoplasmic reticulum homeostasis and calcium mobilization in T lymphocytes. J. Immunol. 186, 1564-1574.

Jounai, N., Takeshita, F., Kobiyama, K. Sawano, A., Miyawaki, A., Xin, K. Q., et al. (2007). The Atg5 Atg12 conjugate associates with innate antiviral immune responses. Proc. Natl. Acad. Sci. U.S.A. 104, 14050-14055.

Jung, H. S., Chung, K. W., Won Kim, J., Kim, J., Komatsu, M., Tanaka, K., et al. (2008). Loss of autophagy diminishes pancreatic beta cell mass and function with resultant hyperglycemia. Cell Metab. 8, 318-324.

Kasai, M., Tanida, I., Ueno, T., Kominami, E., Seki, S., Ikeda, T., et al. (2009). Autophagic compartments gain access to the MHC class II compartments in thymic epithelium. J. Immunol. 183, 7278-7285.

Kaspar, J. W., Niture, S. K., and Jaiswal, A. K. (2009). Nrf2:INrf2 (Keap1) signaling in oxidative stress. Free Radic. Biol. Med. 47, 1304-1309.

Kivity, S., Agmon-Levin, N., Blank, M., and Shoenfeld, Y. (2009). Infections and autoimmunity - friends or foes? Trends Immunol. 30, 409-414.

Komatsu, M., Waguri, S., Koike, M., Sou, Y. S., Ueno, T., Hara, T., et al. (2007) Homeostatic levels of p62 control cytoplasmic inclusion body formation in autophagy-deficient mice. Cell 131, 1149-1163.

Kuballa, P., Nolte, W. M., Castoreno, A. B., and Xavier, R. J. (2012). Autophagy and the immune system. Annu. Rev. Immunol. 30, 611-646.

La Cava, A. (2009). Natural Tregs and autoimmunity. Front. Biosci. 14, 333-343.

Lapaquette, P., Glasser, A. L., Huett, A., Xavier, R. J., and DarfeuilleMichaud, A. (2010). Crohn's diseaseassociated adherent-invasive E. coli are selectively favoured by impaired autophagy to replicate intracellularly. Cell Microbiol. 12, 99-113.

Lee, H. K., Lund, J. M., Ramanathan, B., Mizushima, N., and Iwasaki, A. (2007). Autophagy-dependent viral recognition by plasmacytoid dendritic cells. Science 315, 1398-1401.

Lee, H. K., Mattei, L. M., Steinberg, B. E., Alberts, P., Lee, Y. H., Chervonsky, A., et al. (2010). In vivo requirement for Atg5 in antigen presentation by dendritic cells. Immunity 32 , 227-239.

Levine, B., Mizushima, N., and Virgin, H. W. (2011). Autophagy in immunity and inflammation. Nature 469 323-335.

Lichtman, E. I., Helfgott, S. M., and Kriegel, M. A. (2012). Emerging therapies for systemic lupus erythematosus - focus on targeting interferon-alpha. Clin. Immunol. 143, 210-221.

Lima, J. G., de Freitas Vinhas, C., Gomes, I. N., Azevedo, C. M., dos Santos, R. R., Vannier-Santos, M. A., et al. (2011). Phagocytosis is inhibited by autophagic induction in murine macrophages. Biochem. Biophys. Res. Commun. 405, 604-609.

Maiuri, M. C., Zalckvar, E., Kimchi, A., and Kroemer, G. (2007). Self-eating and self-killing: crosstalk between autophagy and apoptosis. Nat. Rev. Mol. Cell Biol. 8, 741-752.

Manichanh, C., Borruel, N., Casellas, F., and Guarner, F. (2012). The gut microbiota in IBD. Nat. Rev. Gastroenterol. Hepatol. 9, 599-608.

Manjithaya, R., Anjard, C., Loomis, W. F., and Subramani, S. (2010). Unconventional secretion of Pichia pastoris Acbl is dependent on GRASP protein, peroxisomal functions, and autophagosome formation. J. Cell Biol. 188, 537-546.

Martinet, W., Schrijvers, D. M., Timmermans, J. P., Herman, A. G., and De Meyer, G. R. (2009). Phagocytosis of bacteria is enhanced in macrophages undergoing nutrient deprivation. FEBS J. 276, 2227-2240.

Martinez, J., Almendinger, J., Oberst, A., Ness, R., Dillon, C. P., Fitzgerald, P., et al. (2011). Microtubule-associated protein 1 light chain 3 alpha (LC3)associated phagocytosis is required for the efficient clearance of dead cells. Proc. Natl. Acad. Sci. U.S.A. 108, 17396-17401.

McGonagle, D., Aziz, A., Dickie, L. J., and McDermott, M. F. (2009). An integrated classification of pediatric inflammatory diseases, based on the concepts of autoinflammation and the immunological disease continuum. Pediatr. Res. 65, 38R-45R.

Miller, B. C., Zhao, Z., Stephenson, L. M., Cadwell, K., Pua, H. H., Lee, H. K., et al. (2008). The autophagy gene ATG5 plays an essential role in B lymphocyte development. Autophagy 4, 309-314.

Mizushima, N., and Komatsu, M. (2011). Autophagy: renovation of cells and tissues. Cell 147, 728-741.

Mizushima, N., Yamamoto, A., Matsui, M., Yoshimori, T., and Ohsumi, Y. (2004). In vivo analysis of autophagy in response to nutrient starvation using transgenic mice expressing a fluorescent autophagosome marker. Mol. Biol. Cell 15, 1101-1111.

Munz, C. (2009). Enhancing immunity through autophagy. Annu. Rev. Immunol. 27, 423-449.

Nakahira, K., Haspel, J. A., Rathinam, V. A., Lee, S. J., Dolinay, T., Lam, H. C., et al. (2011). Autophagy 
proteins regulate innate immune responses by inhibiting the release of mitochondrial DNA mediated by the NALP3 inflammasome. Nat. Immunol. 12, 222-230.

Nedjic, J., Aichinger, M., Emmerich, J., Mizushima, N., and Klein, L. (2008). Autophagy in thymic epithelium shapes the T-cell repertoire and is essential for tolerance. Nature 455, 396-400.

Ochoa-Reparaz, J., Mielcarz, D. W., Ditrio, L. E., Burroughs, A. R., Foureau, D. M., Haque-Begum, S., et al. (2009). Role of gut commensal microflora in the development of experimental autoimmune encephalomyelitis. J. Immunol. 183, 6041-6050.

Ozcan, U., Yilmaz, E., Ozcan, L., Furuhashi, M., Vaillancourt, E., Smith, R. O., et al. (2006). Chemical chaperones reduce ER stress and restore glucose homeostasis in a mouse model of type 2 diabetes. Science 313, 1137-1140.

Parkes, M., Barrett, J. C., Prescott, N. J., Tremelling, M., Anderson, C. A., Fisher, S. A., et al. (2007). Sequence variants in the autophagy gene IRGM and multiple other replicating loci contribute to Crohn's disease susceptibility. Nat. Genet. 39, 830-832.

Pierdominici, M., Vomero, M., Barbati, C., Colasanti, T., Maselli, A., Vacirca, D., et al. (2012). Role of autophagy in immunity and autoimmunity, with a special focus on systemic lupus erythematosus. FASEB J. 26, 1400-1412.

Pua, H. H., Dzhagalov, I., Chuck, M., Mizushima, N., and He, Y. W. (2007). A critical role for the autophagy gene Atg5 in $\mathrm{T}$ cell survival and proliferation. J. Exp. Med. 204, 25-31.

Pua, H. H., Guo, J., Komatsu, M., and He, Y. W. (2009). Autophagy is essential for mitochondrial clearance in mature T lymphocytes. J. Immunol. 182, 4046-4055.
Qu, X., Zou, Z., Sun, Q., Luby-Phelps, K., Cheng, P., Hogan, R. N., et al. (2007). Autophagy gene-dependent clearance of apoptotic cells during embryonic development. Cell 128, 931-946.

Quan, W., Lim, Y. M., and Lee, M. S. (2012). Role of autophagy in diabetes and endoplasmic reticulum stress of pancreatic beta-cells. Exp. Mol. Med. 44, 81-88.

Randow, F., and Munz, C. (2012). Autophagy in the regulation of pathogen replication and adaptive immunity. Trends Immunol. 33, 475-487.

Raychaudhuri, S., Thomson, B. P., Remmers, E. F., Eyre, S., Hinks, A., Guiducci, C., et al. (2009). Genetic variants at CD28, PRDM1 and $\mathrm{CD} 2 / \mathrm{CD} 58$ are associated with rheumatoid arthritis risk. Nat. Genet. 41, 1313-1318.

Rogers, N. J., Lees, M. J., Gabriel, L., Maniati, E., Rose, S. J., Potter, P. K., et al. (2009). A defect in marco expression contributes to systemic lupus erythematosus development via failure to clear apoptotic cells. J. Immunol. 182, 1982-1990.

Sanjuan, M. A., Dillon, C. P., Tait, S. W., Moshiach, S., Dorsey, F., Connell, S., et al. (2007). Toll-like receptor signalling in macrophages links the autophagy pathway to phagocytosis. Nature 450, 1253-1257.

Schmid, D., and Munz, C. (2007). Innate and adaptive immunity through autophagy. Immunity 27, 11-21.

Schmid, D., Pypaert, M., and Munz, C. (2007). Antigen-loading compartments for major histocompatibility complex class II molecules continuously receive input from autophagosomes. Immunity 26, 79-92.

Shaw, P. J., Lukens, J. R., Burns, S., Chi, H., McGargill, M. A., and Kanneganti, T. D. (2010). Cutting edge: critical role for PYCARD/ASC in the development of experimental autoimmune encephalomyelitis. J. Immunol. 184, 4610-4614.
Shaw, P. J., McDermott, M. F., and Kanneganti, T. D. (2011). Inflammasomes and autoimmunity. Trends. Mol. Med. 17, 57-64.

Sumpter, R. Jr., and Levine, B. (2010) Autophagy and innate immunity: triggering, targeting and tuning. Semin. Cell Dev. Biol. 21, 699-711.

Takeshita, F., Kobiyama, K., Miyawaki, A., Jounai, N., and Okuda, K. (2008). The non-canonical role of Atg family members as suppressors of innate antiviral immune signaling. Autophagy 4, 67-69.

Tal, M. C., Sasai, M., Lee, H. K. Yordy, B., Shadel, G. S., and Iwasaki, A. (2009). Absence of autophagy results in reactive oxygen speciesdependent amplification of RLR signaling. Proc. Natl. Acad. Sci. U.S.A. 106, 2770-2775.

Tang, D., Kang, R., Coyne, C. B., Zeh, H J., and Lotze, M. T. (2012). PAMPs and DAMPs: signal 0s that spur autophagy and immunity. Immunol. Rev. 249, 158-175.

Uhl, M., Kepp, O., Jusforgues-Saklani, H., Vicencio, J. M., Kroemer, G., and Albert, M. L. (2009). Autophagy within the antigen donor cell facilitates efficient antigen cross-priming of virus-specific CD8+ T cells. Cell Death Differ. 16, 991-1005.

Ushio, H., Ueno, T., Kojima, Y., Komatsu, M., Tanaka, S., Yamamoto, A., et al. (2011). Crucial role for autophagy in degranulation of mast cells. J. Allergy Clin. Immunol. 127, 1267.e6-1276.e6.

Watanabe, K., Ichinose, S., Hayashizaki, K., and Tsubata, T. (2008). Induction of autophagy by B cell antigen receptor stimulation and its inhibition by costimulation. Biochem. Biophys. Res. Commun. 374, 274-281.

Wirnsberger, G., Mair, F., and Klein, L. (2009). Regulatory T cell differentiation of thymocytes does not require a dedicated antigen-presenting cell but is under $\mathrm{T}$ cell-intrinsic developmental control. Proc. Natl. Acad. Sci. U.S.A. 106, 10278-10283.
Wu, T., Xie, C., Han, J., Ye, Y., Weiel, J., Li, Q., et al. (2012). Metabolic disturbances associated with systemic lupus erythematosus. PLoS ONE 7:e37210. doi:10.1371/journal.pone.0037210

Zhang, Z., Wu, X., Duan, J., Hinrichs, D., Wegmann, K., Zhang, G. L., et al. (2012). Low dose rapamycin exacerbates autoimmune experimental uveitis. PLoS ONE 7:e36589. doi:10.1371/journal.pone.0036589

Zhou, X. J., Lu, X. L., Lv, J. C., Yang, H. Z., Qin, L. X., Zhao, M. H., et al. (2011a). Genetic association of PRDM1-ATG5 intergenic region and autophagy with systemic lupus erythematosus in a Chinese population. Ann. Rheum. Dis. 70, 1330-1337.

Zhou, R., Yazdi, A. S., Menu, P., and Tschopp, J. (2011b). A role for mitochondria in NLRP3 inflammasome activation. Nature 469, 221-225.

Conflict of Interest Statement: The authors declare that the research was conducted in the absence of any commercial or financial relationships that could be construed as a potential conflict of interest.

Received: 31 January 2013; accepted: 01 April 2013; published online: 15 April 2013.

Citation: Bhattacharya A and Eissa NT (2013) Autophagy and autoimmunity crosstalks. Front. Immunol. 4:88. doi: 10.3389/fimmu.2013.00088

This article was submitted to Frontiers in Antigen Presenting Cell Biology, a specialty of Frontiers in Immunology. Copyright (c) 2013 Bhattacharya and Eissa. This is an open-access article distributed under the terms of the Creative Commons Attribution License, which permits use, distribution and reproduction in other forums, provided the original authors and source are credited and subject to any copyright notices concerning any third-party graphics etc. 